\title{
Molecular Characteristics of Pancreatic Ductal Adenocarcinomas with High-Grade Pancreatic Intraepithelial Neoplasia (PanIN) Are Different from Those without High-Grade PanIN
}

\author{
Tetsuyuki Miyazaki $^{a}$ Yoshihiro Ohishi ${ }^{a}$ Yoshihiro Miyasaka ${ }^{b}$ Yasunori Oda $^{b}$ \\ Shinichi Aishimac Keigo Ozono ${ }^{a}$ Atsushi Abe $^{a}$ Eishi Nagai ${ }^{b}$ \\ Masafumi Nakamurab Yoshinao Oda ${ }^{a}$ \\ Departments of a Anatomic Pathology and ${ }^{\mathrm{b}}$ Surgery and Oncology, Kyushu University, Fukuoka, and ${ }^{\mathrm{C}}$ Department \\ of Pathology and Microbiology, Faculty of Medicine, Saga University, Saga, Japan
}

\section{Keywords}

Pancreatic ductal adenocarcinoma - Pancreatic intraepithelial neoplasia $\cdot$ KRAS $\cdot$ p53 S SMAD4

\begin{abstract}
Aims: We reported that pancreatic ductal adenocarcinomas (PDACs) without high-grade pancreatic intraepithelial neoplasia (PanIN) in the vicinity had worse prognoses than PDACs with high-grade PanIN. However, the molecular characteristics of PDACs with and without high-grade PanIN have not been compared. The aim of this study is to clarify the molecular characteristics of PDACs with and without high-grade PanIN. Method and Results: We reviewed all of a consecutive series of 100 patients with PDACs and divided them into 2 groups: the PDACs with PanIN-2 or PanIN-3 in the background (the PanIN-high group, $n=60$ ) and the PDACs without PanIN-2 or PanIN-3 in the background (the PanIN-low group, $n=40$ ). We evaluated the p53, p16, and SMAD4 expressions in the invasive ductal carcinoma (IDC) components by immunohistochemical staining. KRAS mutation was also analyzed in 80 tumors. The PanIN-low group
\end{abstract}

\section{KARGER}

(C) 2017 S. Karger AG, Basel

E-Mail karger@karger.com

www.karger.com/pat showed significantly more frequent "high p53 expression" and "loss of SMAD4 expression" than the PanIN-high group ( $p=0.048$ and $p=0.019$, respectively). Loss of $\mathrm{p} 16$ expression was not significantly different between the groups. The rate of KRAS wild type was significantly higher in the PanIN-low group than the PanIN-high group $(p=0.024)$. Conclusions: Our results demonstrated that the molecular characteristics in the PDACs with high-grade PanIN were different from those in the PDACs without high-grade PanIN. PDACs without high-grade PanIN may develop via a pathway other than the PanIN-carcinoma sequence.

(c) 2017 S. Karger AG, Basel

\section{Introduction}

Pancreatic ductal adenocarcinoma (PDAC) is one of the most aggressive cancers, with an overall survival rate of $6 \%$ in the USA [1] and 7\% in Japan [2]. Risk factors for PDAC are obesity [3], tobacco smoking [4], heavy alcohol drinking (which is secondary to pancreatitis) [5], and a history of chronic pancreatitis [6]. Patients with the blood

Prof. Yoshinao Oda

Department of Anatomic Pathology Graduate School of Medical Sciences, Kyushu University

3-1-1 Maidashi, Fukuoka 812-8582 (Japan)

E-Mail oda@ @urgpath.med.kyushu-u.ac.jp 
Table 1. Primary antibodies used for immunohistochemical staining

\begin{tabular}{lllll}
\hline Antibody & Clone & Source & Dilution & Antigen retrieval \\
\hline p53 & DO-7 & Novocastra, Newcastle, UK & $1: 100$ & TRS buffer (pH 9.0), microwave 10 min \\
p16 & E6H4 & Roche MTM Laboratories, Heidelberg, Germany & $1: 1$ & PBS (pH 7.4), microwave 20 min \\
Smad4 & B-8 & Santa Cruz Biotechnology, Santa Cruz, CA, USA & $1: 400$ & TRS buffer (pH 9.0), microwave 20 min \\
\hline
\end{tabular}

type A or B are also at higher risk for PDAC than those with type O [7]. Diabetes mellitus is present in $70 \%$ of PDAC patients [8].

Pancreatic intraepithelial neoplasia (PanIN) is well known to be a precursor lesion of PDAC [9]. Less frequently, intraductal papillary mucinous neoplasm and mucinous cystic neoplasm can be precursor lesions of PDAC. PanIN is defined by published consensus guidelines [10]. Briefly, PanINs are microscopic papillary or flat, noninvasive epithelial neoplasms that are usually $<5 \mathrm{~mm}$ in diameter and confined to the pancreatic ducts $[9,10]$. Based on the degree of dysplasia reflected in the cytonuclear atypia and architectural changes, PanINs can be classified into 4 grades: PanIN-1A, $-1 \mathrm{~B},-2$, and -3 $[9,10]$.

Many studies have identified gene alterations of PDAC $[9,11-24]$. One of the major oncogenes, KRAS, is mutated in $>90 \%$ of PDACs $[9,11,21-24]$. In addition, 3 frequently mutated genes play critical roles in the development of pancreatic neoplasias: CDKN2A (p16), TP53 and SMAD4 (DPC4) [9, 12-27]. The p16, TP53, and SMAD4 genes are inactivated in $95,50-75$, and $55 \%$ of PDACs, respectively [9, 12-24]. In PanIN lesions, KRAS activations appear to occur relatively early; $p 16$ inactivation occurs at an intermediate stage, and $p 53$ and SMAD4 inactivations occur at a late stage [9, 13, 24-27].

We previously demonstrated that PDAC patients without PanIN-2 or PanIN-3 in the background (i.e., the PanIN-low group) had worse prognoses than those with PanIN-2 or PanIN-3 (the PanIN-high group) [28]. However, the mechanisms underlying the poor prognosis in the PanIN-low group remained unclear. In the present study we examined and compared the molecular characteristics of a PanIN-low group and a PanIN-high group.

\section{Materials and Methods}

\section{Patients and Specimens}

We retrospectively reviewed samples from 100 PDAC cases that had been diagnosed at the Department of Anatomic Pathology of Kyushu University (Fukuoka, Japan) between January 1992 and December 2009. Eighty of the 100 patients with PDAC were included in our previous study [28]. Clinical and pathological data were obtained from a retrospective database and a review of medical records. Clinical characteristics (age, sex, risk factors, diabetes mellitus, symptoms, body mass index, and tumor location) and pathological findings (lymphatic permeation, venous invasion, perineural invasion, histological grade, and UICC TNM stage) were evaluated. Alcohol intake was estimated as described [5]. Survival was measured from the time point of the surgery until death or censor. This study was approved by the Ethics Committee of Kyushu University (No. 27-242).

\section{Pathological Examination for PanIN}

The surgically resected specimens were fixed with formalin in approximately 5-mm-thick sections. All of the consecutive whole tissue sections of all resected pancreatic tissue including tumor lesions were embedded in paraffin and routinely examined microscopically with hematoxylin and eosin (HE) staining. The average number of investigated slides per case was 22.2 (range 11-44). All of the investigated slides were taken from the pancreatic tissue including the tumor lesion, and the slides taken from nonpancreatic tissue (lymph nodes, nonpancreatic margins, etc.) were not included and counted. Three pathologists (S.A., Y. Oda, and T.M.) independently evaluated PanINs that were $<5 \mathrm{~mm}$ in size within the pancreatic duct and those that were distinctly separate from the invasive carcinoma component. We evaluated PanIN lesions accompanied by normal pancreatic tissue between PanIN lesions and the invasive ductal carcinoma (IDC) component. We histologically defined "PanIN-3" as an intraductal neoplastic lesion showing severe cellular atypia, a loss of cell polarity, and a micropapillary structure, accompanied by the close proximity of PanIN1A, PanIN-1B, or PanIN-2, and no involvement by an invasive carcinoma component. It was necessary for there to be at least 5 $\mathrm{mm}$ of normal pancreas between the PanIN-3 and the cancer. Cancerization of the duct, which is defined as carcinoma secondarily spreading into the pancreatic duct, was excluded from PanIN-3.

We counted the numbers of PanIN lesions (i.e., the numbers of PanIN-1A, PanIN-1B, PanIN-2, and PanIN-3 lesions, respectively) in each IDC. The median number of total PanIN lesions, regardless of the grade of dysplasia, was 15 (range 0-595). We classified the lesions into a $<15$ PanIN group $(n=45)$ and $a \geq 15$ PanIN group $(n=55)$. We retrospectively investigated all slides and recorded the number of slides and the grade of all PanIN lesions in all samples.

Because PanINs can have various grades in the same samples, we classified the 100 patients with PDACs into a PanIN-low group (the PDACs with PanIN-1A, -1B or the absence of PanIN) and a PanIN-high group (the PDACs with PanIN-2 or PanIN-3) based on the highest grade of atypia. The detailed evaluation method for

Molecular Characteristics of PDACs with

Pathobiology 2017;84:192-201 
Table 2. Correlations between clinicopathological characteristics and PanIN group in PDACs

\begin{tabular}{|c|c|c|c|}
\hline & \multicolumn{2}{|c|}{ PanIN group } & \multirow[t]{2}{*}{$p$ value } \\
\hline & $\begin{array}{l}\text { low } \\
(n=40)\end{array}$ & $\begin{array}{l}\text { high } \\
(n=60)\end{array}$ & \\
\hline \multicolumn{4}{|l|}{ Age } \\
\hline$<65$ years & 16 & 25 & \multirow[t]{2}{*}{0.868} \\
\hline$\geq 65$ years & 24 & 35 & \\
\hline Male:female & $27: 13$ & $33: 27$ & 0.211 \\
\hline \multicolumn{4}{|l|}{ Location } \\
\hline Head:body/tail & $26: 14$ & $42: 18$ & 0.599 \\
\hline \multicolumn{4}{|l|}{ Family history } \\
\hline Present/absent & $35 / 1$ & $53 / 3$ & \multirow[t]{2}{*}{$1.000^{\mathrm{a}}$} \\
\hline Unknown & 4 & 4 & \\
\hline \multicolumn{4}{|l|}{ Symptoms } \\
\hline Present/absent & $11 / 29$ & $24 / 36$ & 0.199 \\
\hline \multicolumn{4}{|l|}{ Smoking history } \\
\hline Yes/no & $23 / 13$ & $36 / 21$ & \multirow[t]{2}{*}{0.943} \\
\hline Unknown & 4 & 3 & \\
\hline \multicolumn{4}{|l|}{ Drinking } \\
\hline Non- or occasional & 22 & 39 & \multirow[t]{4}{*}{0.578} \\
\hline Moderate & 3 & 6 & \\
\hline Heavy & 11 & 12 & \\
\hline Unknown & 4 & 3 & \\
\hline $\mathrm{BMI}<25 / \geq 25$ & $31 / 6$ & $53 / 3$ & 0.083 \\
\hline Unknown & 3 & 4 & \\
\hline \multicolumn{4}{|l|}{ ABO blood group } \\
\hline O/others & $6 / 30$ & $13 / 43$ & \multirow[t]{2}{*}{0.449} \\
\hline Unknown & 4 & 4 & \\
\hline \multicolumn{4}{|l|}{ Pancreatitis history } \\
\hline Present/absent & $4 / 33$ & $3 / 55$ & \multirow[t]{2}{*}{0.305} \\
\hline Unknown & 3 & 2 & \\
\hline \multicolumn{4}{|l|}{ Diabetes mellitus } \\
\hline Present/absent & $33 / 4$ & $48 / 9$ & \multirow[t]{2}{*}{0.495} \\
\hline Unknown & 3 & 3 & \\
\hline \multicolumn{4}{|l|}{ Histological grade } \\
\hline G1 & 13 & 22 & \multirow[t]{3}{*}{0.350} \\
\hline G2 & 18 & 31 & \\
\hline G3 & 9 & 7 & \\
\hline Vessel invasion (+) & $33(82.5 \%)$ & $34(56.7 \%)$ & $0.007^{*}$ \\
\hline Lymphatic permeation $(+)$ & $36(90 \%)$ & $43(71.7 \%)$ & $0.027^{*}$ \\
\hline Perineural invasion $(+)$ & $36(90 \%)$ & $53(88.3 \%)$ & 0.794 \\
\hline \multicolumn{4}{|l|}{ UICC TNM category } \\
\hline $\mathrm{T} 1+2 / 3+4$ & $2 / 38$ & $5 / 55$ & $0.699^{\mathrm{a}}$ \\
\hline N0/N1 & $11 / 29$ & $20 / 40$ & 0.537 \\
\hline $\mathrm{M} 0 / \mathrm{M} 1$ & $38 / 2$ & $59 / 1$ & $0.562^{\mathrm{a}}$ \\
\hline \multicolumn{4}{|l|}{ UICC stage } \\
\hline $\mathrm{I}+\mathrm{II} / \mathrm{III}+\mathrm{IV}$ & $37 / 3$ & $58 / 2$ & $0.386^{\mathrm{a}}$ \\
\hline \multicolumn{4}{|l|}{ Number of PanIN lesions } \\
\hline$\geq 15$ PanINs/<15 PanINs & $13 / 27$ & $42 / 18$ & $0.0002^{*}$ \\
\hline
\end{tabular}

* Statistically significant. ${ }^{a}$ Fisher's exact tests.
PanIN in the background has previously been described [28]. We also determined the correlations between the PanIN groups and clinicopathological findings.

\section{Immunohistochemical Procedures}

The primary monoclonal antibodies used for the immunohistochemical analysis are listed in Table 1 . In brief, 4 - $\mu$ m-thick tissue sections were deparaffinized in xylene and dehydrated in ethanol. Endogenous peroxidase activity was blocked by incubation in methanol containing $0.3 \% \mathrm{H}_{2} \mathrm{O}_{2}$ for $30 \mathrm{~min}$. Antigen retrieval was achieved as shown in Table 1.

The slides were then incubated with the primary antibodies for $90 \mathrm{~min}$ at room temperature (p53 and p16) or at $4^{\circ} \mathrm{C}$ overnight (SMAD4) and subsequently incubated with biotin-free horseradish peroxidase enzyme-labeled polymer (EnVision plus System, DAKO Cytomation, Glostrup, Denmark) for $40 \mathrm{~min}$ at room temperature. The labeled antigens were visualized using 3,3' -diaminobenzidine tetrahydrochloride as a chromogen. Counterstaining was performed with hematoxylin. Appropriate positive and negative controls were performed for all antibodies.

\section{Evaluation of Immunohistochemical Staining}

We analyzed both 100 IDCs and 95 concomitant PanIN components. Immunohistochemical staining of 5 of the PanIN lesions was not available because 5 PanIN lesions disappeared after the serial sectioning of the paraffin blocks. Consequently, 90 PanIN components were evaluated by immunohistochemical staining. The reactions were interpreted as positive on the basis of the presence of nuclear staining (p53) and nuclear and cytoplasmic staining (p16 and SMAD4).

For the p53 immunolabeling, focal acinar and ductal cells with nuclear p53 immunolabeling served as an internal control. The p53 immunolabeling was interpreted as "high expression" when the neoplastic cells showed robust nuclear accumulation in $\geq 30 \%$ of the neoplastic cells. Immunohistochemical labeling of high p53 expression corresponded to mutational inactivation [23, 29-31].

The immunohistochemical labeling of p16 and SMAD4 was defined as intact (positive) or completely lost (negative). Negative p16 and SMAD4 immunolabeling is explained by gene alterations such as homozygous deletion, promoter hypermethylation or loss of heterozygosity and intragenic mutation [16, 20,29]. Islet cells served as an internal control for positive p16 immunolabeling. Normal acinar cells, ductal cells, islet cells, and stromal cells in each case served as internal controls for positive SMAD4 immunolabeling.

\section{Mutational Analysis of the IDC Component}

In the 100 PDACs, genomic DNA was extracted from 5 or six 7 - $\mu \mathrm{m}$ sections of formalin-fixed, paraffin-embedded specimens by a macrodissection technique. We carefully macrodissected from portions containing as many carcinoma cells as possible, referring to the HE slides. The range of the percentage of carcinoma cells within the macrodissected tissue was $30-50 \%$. Genomic DNA was subsequently extracted using the QIAamp DNA FFPE Tissue Kit according to the manufacturer's instructions (Qiagen, Hilden, Germany). The polymerase chain reaction (PCR) and mutational analysis were performed for exon 2 of KRAS.

For 16 cases (high and low levels of p53 immunohistochemical expressions in 8 cases each), we also performed a mutational analysis of the TP53 gene (exons 5-9). 

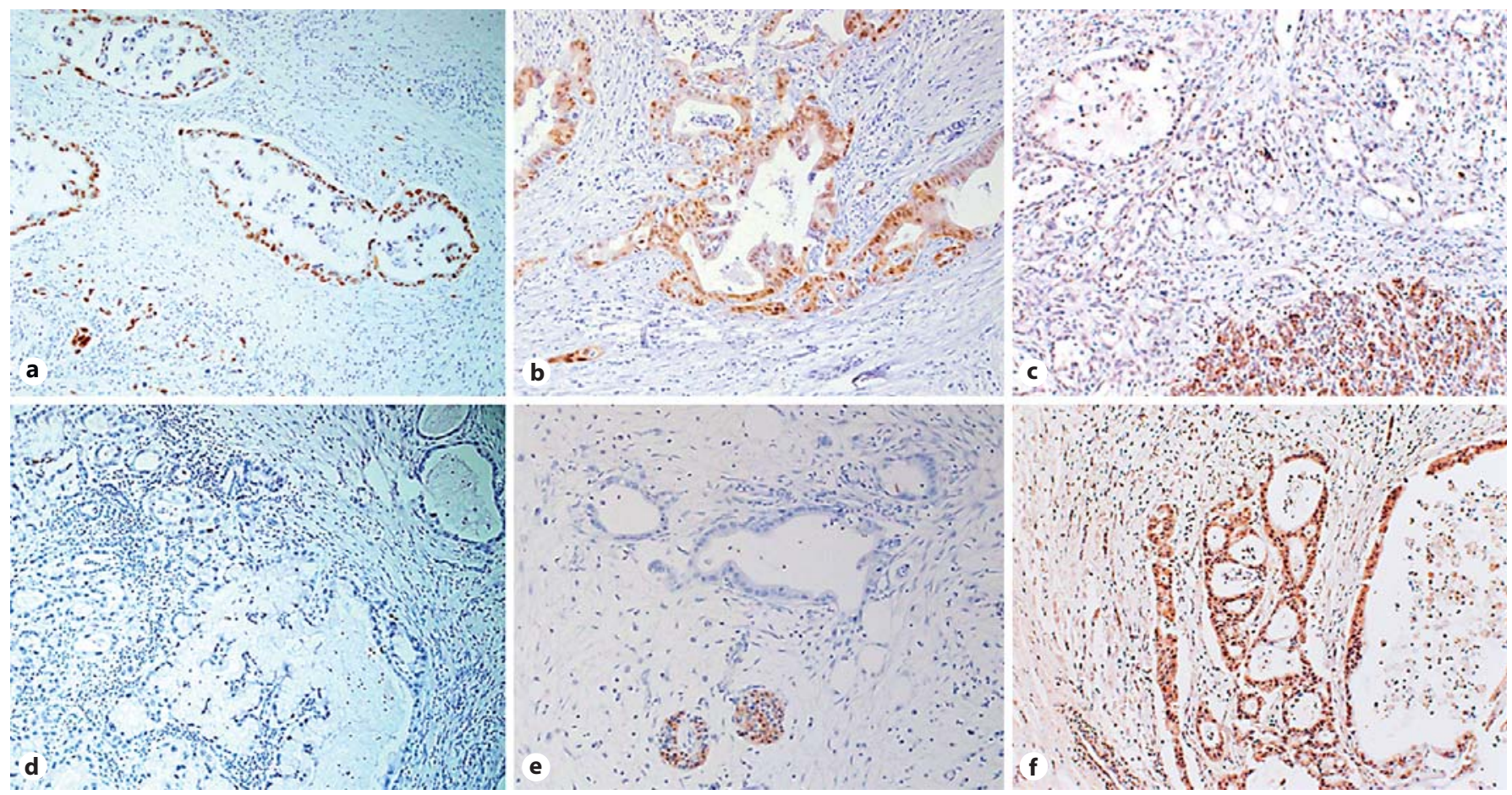

Fig. 1. Representative immunohistochemical images of p53, p16, and SMAD4 expression in the IDC component of the PanIN-low (a-c) and PanIN-high (d-f) groups. High p53 expression (a), p16 intact (b), and SMAD4 loss (c) were observed in the PanIN-low group, whereas low p53 expression (d), p16 loss (e), and SMAD4

intact (f) were observed in the PanIN-high group. p16 expression was preserved in normal islet cells (e, below). SMAD4 expression was preserved in normal acinar cells (c, below). a-f Original magnification, $\times 100$.

Table 3. Correlation between PanIN groups for p53, p16, and SMAD4 expression in the IDC component

\begin{tabular}{|c|c|c|c|}
\hline & \multicolumn{2}{|l|}{ PanIN group } & \multirow[t]{2}{*}{$p$ value } \\
\hline & low $(n=40)$ & $\operatorname{high}(n=60)$ & \\
\hline \multicolumn{4}{|l|}{ p53 } \\
\hline Low expression & 24 & 47 & \multirow[t]{2}{*}{$0.048^{*}$} \\
\hline High expression & $16(40 \%)$ & $13(21.7 \%)$ & \\
\hline \multicolumn{4}{|l|}{ p16 } \\
\hline Positive (intact) & 14 & 11 & \multirow[t]{2}{*}{0.059} \\
\hline Negative (loss) & $26(65 \%)$ & $49(81.7 \%)$ & \\
\hline \multicolumn{4}{|l|}{ SMAD4 } \\
\hline Positive (intact) & 10 & 29 & \multirow[t]{2}{*}{$0.019^{*}$} \\
\hline Negative (loss) & $30(75 \%)$ & $31(51.7 \%)$ & \\
\hline
\end{tabular}

* Statistically significant.

The primer pairs used in the sequencing analysis are shown in the online supplementary Table 1 (for all online suppl. material, see www.karger.com/doi/10.1159/000455194).

The amplified PCR products were then purified using Montage centrifugal filters (Millipore, Bedford, MA, USA). After purifica-

Molecular Characteristics of PDACs with and without High-Grade PanIN tion, direct sequencing was carried out using a Perkin Elmer ABI PRISM 310 sequence analyzer (Applied Biosystems, Foster City, CA, USA). If the quality of DNA or the level of PCR amplification was insufficient for the mutation analysis, the cases were excluded from the molecular study.

\section{Statistical Analysis}

All calculations were carried out using JMP 11.0.0 software (SAS Institute, Cary, NC, USA). Statistical analyses were performed with the Fisher exact test, the $\chi^{2}$ test and the Mann-Whitney U test. Survival curves were examined by the Kaplan-Meier method, and significance was examined using the log-rank test. Multivariate survival analyses were performed using the Cox proportional hazards regression model. A $p$ value of $<0.05$ was considered significant.

\section{Results}

Frequency of PanIN Grade and the Classification of the PanIN Groups

According to the highest grade of PanIN, the 100 patients were classified as follows: no PanIN $(n=5)$, PanIN-1A $(n=7)$, PanIN-1B $(n=28)$, PanIN-2 $(n=38)$, 
Table 4. Rate of p53, p16, and SMAD4 expression in PanIN and the IDC component of the PanIN group

\begin{tabular}{|c|c|c|c|c|c|c|}
\hline \multirow[t]{2}{*}{ p53 } & \multicolumn{3}{|c|}{ PanIN-low group } & \multicolumn{3}{|c|}{ PanIN-high group } \\
\hline & PanIN-1A & PanIN-1B & IDC & PanIN-2 & PanIN-3 & IDC \\
\hline High expression & $0 \%(0 / 7)$ & $0 \%(0 / 28) *$ & $40 \%(16 / 40)$ & $2.9 \%(1 / 35)$ & $15 \%(3 / 20)$ & $21.7 \%(13 / 60)$ \\
\hline p16 loss & $28.6 \%(2 / 7)$ & $17.9 \%(5 / 28) *$ & $65 \%(26 / 40)$ & $28.6 \%(10 / 35)$ & $55 \%(11 / 20)^{*}$ & $81.7 \%(49 / 60)$ \\
\hline SMAD4 loss & $14.3 \%(1 / 7)$ & $0 \%(0 / 28)^{*}$ & $75 \%(30 / 40)$ & $5.7 \%(2 / 35)$ & $25 \%(5 / 20)^{*}$ & $51.7 \%(31 / 60)$ \\
\hline
\end{tabular}

$* p \leq 0.05:$ statistically significantly different compared to IDC.

and PanIN-3 $(n=22)$. We categorized the patients into the PanIN-low group $(n=40)$ and the PanIN-high group $(n=60)$. There was no significant difference in the number of HE-stained slides reviewed between the 2 groups.

\section{Correlations between the 2 PanIN Groups and \\ Clinicopathological Characteristics}

We compared the correlations between the 2 PanIN groups and the patients' clinicopathological characteristics (Table 2). The PanIN-low group showed significantly more frequent vessel invasion, lymphatic permeation and a smaller number of PanIN lesions compared to the PanIN-high group ( $p=0.007, p=0.027$, and $p=0.0002$, respectively). There were no significant relationships between the PanIN group and the other clinicopathological characteristics.

The Expressions of p53, p16, and SMAD4 in the IDC Component: Comparison of the Low-and High-PanIN Groups

The IDC component immunohistochemical results are summarized in Table 3. Representative immunohistochemical images are shown in Figure 1. The frequency of high p53 expression was significantly higher in the PanIN-low group compared to the PanIN-high group (40 vs. $22 \%, p=0.048$ ). Loss of SMAD4 expression was observed significantly more frequently in the PanIN-low group compared to the PanIN-high group (75 vs. 52\%, $p=0.019)$. The frequency of loss of p16 expression tended to be low in the PanIN-low group compared to the PanIN-high group, but there was no significant difference ( 65 vs. $81.7 \%, p=0.059$ ).

\section{p53, p16, and SMAD4 Expression in PanIN and IDC} Components

The immunohistochemical results of PanIN and the IDC components in the PanIN-low group are summa-
Table 5. Correlation between PanIN group and KRAS status in the IDC component

\begin{tabular}{llll}
\hline KRAS mutation & \multicolumn{2}{l}{ PanIN group } & $\begin{array}{l}p \\
\text { value }\end{array}$ \\
\cline { 2 - 3 } & low $(n=33)$ & high $(n=47)$ & \\
\hline- & $14(42.4 \%)$ & $9(19.2 \%)$ & $0.024^{*}$ \\
+ & $19(57.6 \%)$ & $38(80.8 \%)$ & \\
\hline
\end{tabular}

* Statistically significant.

rized in Table 4 . The rates of high p53 expression were 0 , 0 , and $40 \%$ in the PanIN-1A, PanIN-1B, and IDC components, respectively. The rates of loss of p16 expression were 28.6, 17.9, and 65\% in the PanIN-1A, PanIN-1B, and IDC components, respectively. The rates of loss of SMAD4 expression were $14.3,0$, and $75 \%$ in the PanIN-1A, PanIN$1 \mathrm{~B}$, and IDC components, respectively. In the PanIN-low group, the rates of p53 high expression, loss of p16 expression and loss of SMAD4 expression were very low in the low-grade PanIN components but were notably high in the IDC component $(p=0.0001, p=0.0001$, and $p<$ 0.0001 , respectively).

For the PanIN-high group, the immunohistochemical results of PanIN and the IDC components are summarized in Table 4 . The rates of high p53 expression were $2.9,15$, and $21.7 \%$ in the PanIN-2, PanIN-3, and IDC components, respectively. The rates of loss of p16 expression were 28.6, 55, and $81.7 \%$ in the PanIN-2, PanIN-3, and IDC components, respectively. The rates of loss of SMAD4 expression were 5.7, 25, and $51.7 \%$ in the PanIN-2, PanIN-3, and IDC components, respectively. In the PanIN-high group, the rates of p53 high expression, loss of p16 expression, and loss of SMAD4 expression were gradually increased as the lesions progressed to PanIN-2, PanIN-3, and IDC (Table 4).
196

Pathobiology 2017;84:192-201 DOI: $10.1159 / 000455194$
Miyazaki/Ohishi/Miyasaka/Oda/Aishima/ Ozono/Abe/Nagai/Nakamura/Oda 


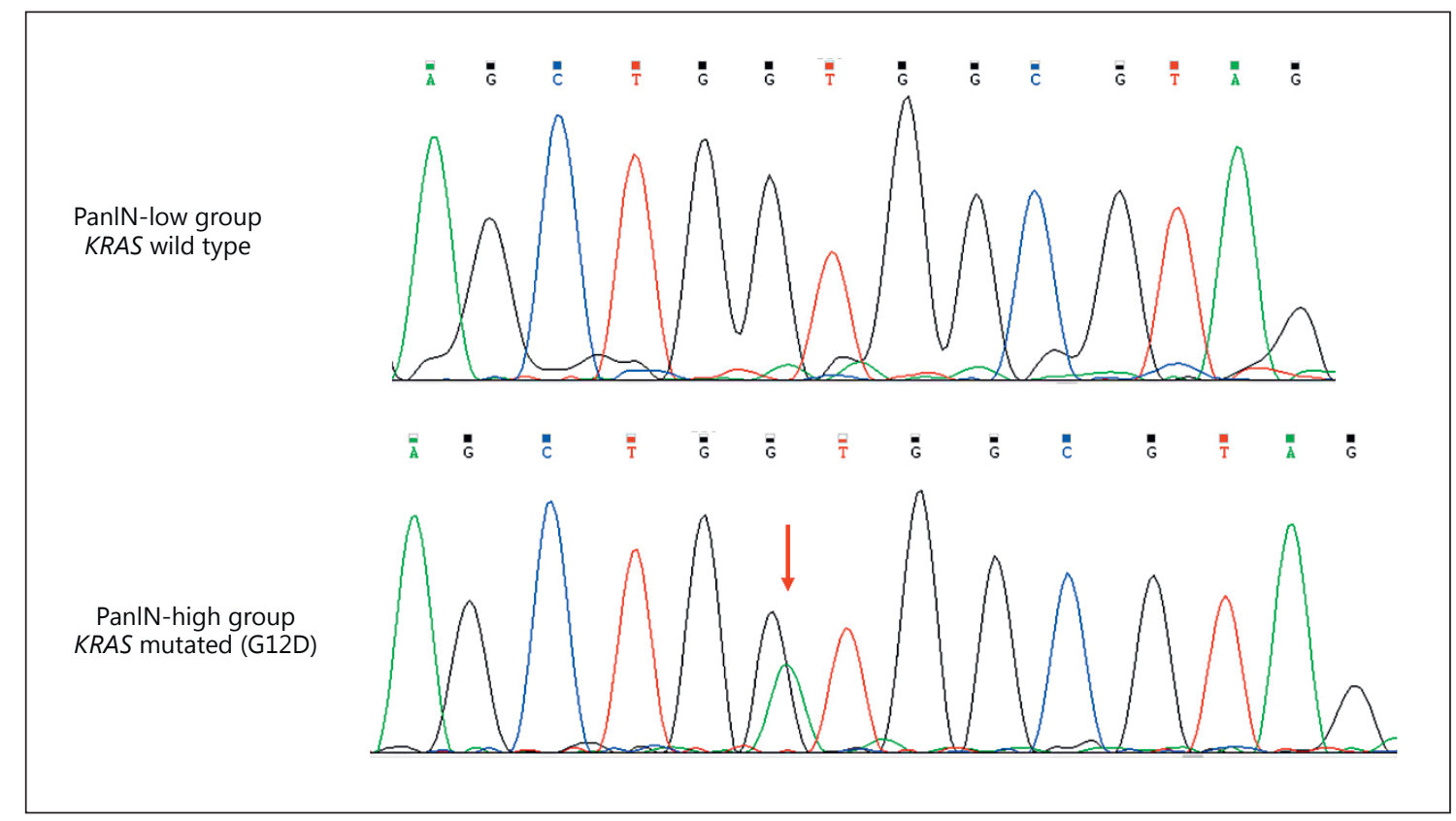

Fig. 2. Representative sequencing results of KRAS wild type in the PanIN-low group and KRAS mutation in the PanIN-high group. KRAS wild type was noted in the PanIN-low group, whereas KRAS mutation (G12D) was seen in the PanIN-high group.

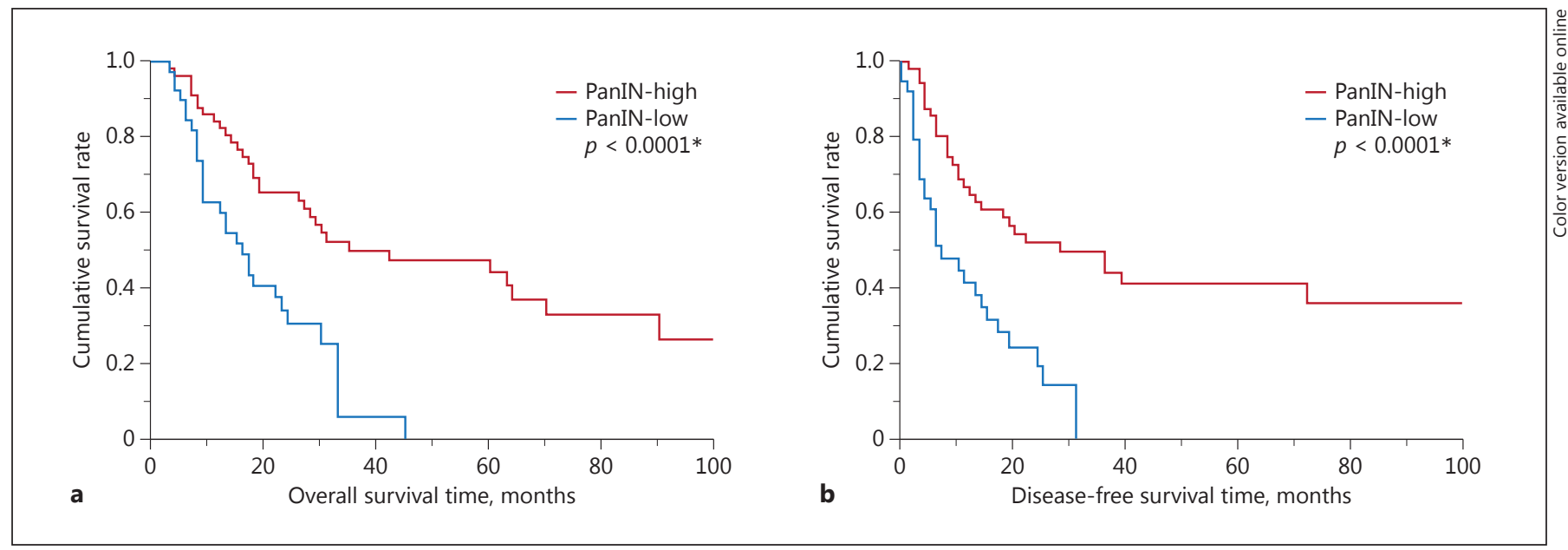

Fig. 3. Survival analysis of patients in the PanIN-low and PanIN-high groups. Overall survival (a) and diseasefree survival (b) were both significantly shorter in the PanIN-low group compared to the PanIN-high group ( $p$ $<0.0001$, respectively).

\section{KRAS Mutational Analysis}

The results of the KRAS mutational analysis are summarized in Table 5 and Figure 2. Eighty of the 100 cases were successfully sequenced. The remaining 20 cases were excluded from the KRAS mutation analysis because of an insufficient quality of DNA or level of PCR ampli- fication. KRAS wild type was noted in $14 / 33$ (42.4\%) of the PanIN-low group and in 9/47 (19.2\%) of the PanINhigh group. The rate of KRAS wild type was significantly higher in the PanIN-low group compared to the PanINhigh group $(p=0.024)$. 


\section{TP53 Mutational Analysis}

The results of the TP53 gene mutational analysis are summarized in supplementary Table 2 in relation to the level of immunohistochemical expressions of $\mathrm{p} 53$ and the PanIN group. TP53 gene mutation was observed in only 2 cases with high immunohistochemical expression of p53, and both cases were in the PanIN-low group.

\section{Survival Analysis}

Disease-free and overall survivals were significantly shorter in the PanIN-low group compared to the PanINhigh group (Fig. $3 ; p<0.0001$ and $p<0.0001$, respectively). The median survival times for the PanIN-low group and the PanIN-high group were 16 and 42 months, respectively. Regarding the number of PanIN lesions, overall survival was significantly longer in the $\geq 15$ PanIN group than in the $<15$ PanIN group (online suppl. Fig. 3; $p=0.018$ ). There were no significant differences in disease-free survival between the $\geq 15$ PanIN group and the $<15$ PanIN group (online suppl. Fig. 3; $p=0.059$ ). The univariate survival analysis indicated that smoking history, heavy drinking, histological grade, lymphatic permeation, vessel invasion, lymph node metastasis, being in the PanIN-low group and the loss of SMAD4 expression were significant worse-prognosis factors (Table 6).

The multivariate survival analysis based on the Cox proportional hazards model included all parameters found to be significant in the univariate analyses and well-known prognosis factors such as UICC TNM stage and perineural invasion [9]. The multivariate survival analysis revealed that heavy drinking, lymph node metastasis and being in the PanIN-low group were independent prognostic factors (Table 6).

\section{Discussion}

There have been a few reports demonstrating the correlation between the grade of coexisting PanIN around IDC and prognosis $[28,32]$. We observed previously that PDACs without high-grade PanIN led to a worse prognosis [28]. Hassid et al. reported that their patients who demonstrated an absence of PanIN in the pancreatic tissue adjacent to the resected PDAC tumor had shorter postresection survivals compared to those who demonstrated a PanIN lesion [32]. However, the molecular characteristics of PDACs based on the grade of concomitant PanIN have not been well investigated. This is the first report on differences in molecular characteristics between PDACs with and without high-grade PanIN.
We also observed that the rates of high p53 expression and loss of SMAD4 expression were significantly higher in the PanIN-low group compared to the PanIN-high group, whereas the rate of loss of p16 expression tended to be high in the PanIN-high group compared with the PanIN-low group, though not significantly (Table 3 ). It has been reported that the immunohistochemical labeling of p53, p16 and SMAD4 reflects the genetic status of TP53, p16 and SMAD4 [16, 20, 23, 29-31]. Thus, there may be high frequencies of TP53 mutation and inactivation of the SMAD4 gene in PanIN-low patients compared to the PanIN-high group. On the other hand, there may be a high frequency of inactivation of the $p 16$ gene in the PanIN-high group compared to the PanIN-low group.

In our PanIN-high group, the rates of high p53 expression, loss of p16 expression and loss of SMAD4 expression were increased stepwise from PanIN to IDC (Table 4). In the PanIN-low group, the rates of high p53 expression, loss of p16 expression and loss of SMAD4 expression in the PanIN component were very low, but much higher in the IDC component (Table 4). These results are consistent with previous studies $[9,12-14,17-$ $19,24,26,27]$.

In this study, TP53 gene mutation was detected in only 2 of the 16 cases examined, probably because the quality of extracted DNA was insufficient for a sequencing analysis of all of the exons of the TP53 gene. We thus could not obtain further clarification regarding a direct relationship between TP53 gene status and PanIN groups. However, the fact that both cases with TP53 gene mutations showed a high immunohistochemical expression of p53 was in line with our supposition that immunohistochemical high expression corresponds to mutational inactivation [29-32].

Inactivation of $p 16$ in pancreatic adenocarcinomas is caused by homozygous deletion (40\%), intragenic mutation in 1 allele coupled with loss of the second allele (loss of heterozygosity) (40\%), and hypermethylation of a CpG island in the promoter region $(15 \%)[14,15]$. Inactivation of SMAD4 in pancreatic adenocarcinomas is caused by homozygous deletion (35\%) and loss of heterozygosity (25\%) [17-19].

Immunohistochemical labeling for the p16 and SMAD4 proteins has been shown to mirror these genes' status $[16,20]$. This provides a simple method to estimate these genes' status in resected tissues. We thus chose this indirect immunohistochemical method because it was shown that immunohistochemistry for p16 and SMAD4 is a surrogate marker for these genes' status [16, 20]. However, we did not directly analyze genetic and epigen-
198

Pathobiology 2017;84:192-201

DOI: $10.1159 / 000455194$
Miyazaki/Ohishi/Miyasaka/Oda/Aishima/ Ozono/Abe/Nagai/Nakamura/Oda 
Table 6. Univariate and multivariate analyses of overall survival of the PDAC patients

\begin{tabular}{|c|c|c|c|c|}
\hline & \multirow{2}{*}{$\begin{array}{l}\text { Univariate analysis } \\
p \text { value }\end{array}$} & \multicolumn{3}{|c|}{ Multivariate analysis } \\
\hline & & hazard ratio & $\begin{array}{l}95 \% \text { confidence } \\
\text { interval }\end{array}$ & $p$ value \\
\hline \multicolumn{5}{|l|}{ Sex } \\
\hline Male/female & 0.0943 & & & \\
\hline \multicolumn{5}{|l|}{ Age } \\
\hline$\geq 65$ years $/<65$ years & 0.8801 & & & \\
\hline \multicolumn{5}{|l|}{ Location } \\
\hline Head/body and tail & 0.5011 & & & \\
\hline \multicolumn{5}{|l|}{ Family history } \\
\hline Present & 0.6732 & & & \\
\hline \multicolumn{5}{|l|}{ Symptoms } \\
\hline Present & 0.3707 & & & \\
\hline \multicolumn{5}{|l|}{ Smoking history } \\
\hline Yes & $0.0287^{*}$ & 1.253 & $0.579-2.689$ & 0.563 \\
\hline \multicolumn{5}{|l|}{ Drinking } \\
\hline Heavy/non- or occasional & $0.0003^{*}$ & 3.655 & $1.674-7.943$ & $0.0012^{*}$ \\
\hline Heavy/moderate & $0.0105^{*}$ & 2.956 & $1.114-8.881$ & $0.0287^{*}$ \\
\hline Moderate/non- or occasional & 0.9752 & & & \\
\hline \multicolumn{5}{|l|}{ BMI } \\
\hline$\geq 25 /<25$ & 0.873 & & & \\
\hline \multicolumn{5}{|l|}{ ABO blood group } \\
\hline The others/O & 0.140 & & & \\
\hline \multicolumn{5}{|l|}{ Pancreatitis history } \\
\hline Present & 0.0568 & & & \\
\hline \multicolumn{5}{|l|}{ Diabetes mellitus } \\
\hline Present & 0.5466 & & & \\
\hline \multicolumn{5}{|l|}{ Histological grade } \\
\hline $\mathrm{G} 3 / \mathrm{G} 1$ & $0.0086^{*}$ & 1.201 & $0.468-3.083$ & 0.700 \\
\hline $\mathrm{G} 2 / \mathrm{G} 1$ & 0.2356 & & & \\
\hline \multicolumn{5}{|l|}{ Lymphatic permeation } \\
\hline Positive & $0.0005^{*}$ & 1.563 & $0.630-4.347$ & 0.345 \\
\hline \multicolumn{5}{|l|}{ Vessel invasion } \\
\hline Positive & $<0.0001^{*}$ & 2.063 & $0.937-4.844$ & 0.072 \\
\hline \multicolumn{5}{|l|}{ Perineural invasion } \\
\hline Positive & 0.9053 & 0.583 & $0.218-1.782$ & 0.325 \\
\hline \multicolumn{5}{|l|}{ UICC pT category } \\
\hline $\mathrm{T} 3+4$ & 0.2151 & 0.483 & $0.105-2.678$ & 0.380 \\
\hline \multicolumn{5}{|l|}{ UICC $\mathrm{pN}$ category } \\
\hline N1 & $0.0004^{*}$ & 3.261 & $1.451-7.873$ & $0.003^{*}$ \\
\hline \multicolumn{5}{|l|}{ UICC pM category } \\
\hline M1 & 0.0661 & 0.000 & $0-3.827$ & 0.286 \\
\hline \multicolumn{5}{|l|}{ PanIN group } \\
\hline Low & $0.0002^{*}$ & 2.096 & $1.085-4.104$ & $0.027^{*}$ \\
\hline \multicolumn{5}{|l|}{ p53 } \\
\hline High & 0.1339 & & & \\
\hline p16 & & & & \\
\hline Loss & 0.845 & & & \\
\hline SMAD4 & & & & \\
\hline Loss & $0.0461^{*}$ & 1.466 & $0.780-2.832$ & 0.236 \\
\hline
\end{tabular}

* Statistically significant. 
etic alterations of $p 16$ and SMAD4 genes in the present study, and we could not validate our immunohistochemical data. Further genetic and epigenetic analyses may be necessary in the future.

In studies of ovarian serous and colorectal carcinomas, the carcinomas without a precursor lesion had worse prognoses and usually the KRAS wild type [33, 34]. It has been proposed that these carcinomas may arise via a de novo pathway rather than an adenoma-carcinoma sequence $[33,34]$. In the present study, there was a high rate of KRAS wild type in the PanIN-low group (Table 5), and thus the PanIN-low group was more likely to include tumors that develop via a distinct pathway other than the PanIN-carcinoma sequence. Hassid et al. [32] noted that the subset of tumors lacking PanIN may represent a biologically distinct group arising independently from the usual PanIN route in an as yet undefined pathway. Some cases of the PanIN-low group in our study may be included in such a biologically distinct group.

Vascular and lymphatic invasions, which are well known as poor prognostic factors for pancreatic cancer $[9,29]$, were more frequent in the PanIN-low group compared to the PanIN-high group (Table 2). This may be 1 reason for the poor prognosis of the PanIN-low group.
Our multivariate survival analysis showed that being in the PanIN-low group was an independent prognostic factor (Table 6). The carcinogenic pathway of the PanIN-low and PanIN-high groups may correspond to "de novo" and "adenoma-carcinoma" sequences, respectively.

In conclusion, the molecular characteristics in some PDACs without high-grade PanIN may be different from those of the PDACs with high-grade PanIN. There is a possibility that some PDACs without high-grade PanIN may develop via a pathway other than the PanIN-carcinoma sequence.

\section{Acknowledgments}

We appreciate Takeshi Iwasaki, Mami Nakamizo, and the technical assistance from the Research Support Center, Research Center for Human Disease Modeling, Kyushu University Graduate School of Medical Sciences.

\section{Disclosure Statement}

The authors declare that there are no conflicts of interest to disclose.

\section{References}

1 Siegel R, Naishadham D, Jemal A: Cancer statistics, 2013. CA Cancer J Clin 2013;63:11-30.

2 Wakao F, Nishimoto H, Katanoda K, et al (eds): 5-Year Relative Rate (7 Registries), Both Sexes. Cancer Statistics in Japan 2014. Tokyo, Foundation for Promotion of Cancer Research, 2015, p 80.

3 Marmot M, Atinmo T, Byers T, et al (eds): Cancers. World Cancer Research Fund/ American Institute for Cancer Research. Food, Nutrition, Physical Activity, and the Prevention of Cancer: A Global Perspective. Washington, American Institute for Cancer Research, 2007, pp 271-274.

4 Iodice S, Gandini S, Maisonneuve P, et al: Tobacco and the risk of pancreatic cancer: a review and meta-analysis. Langenbecks Arch Surg 2008;393:535-545.

5 Tramacere I, Scotti L, Jenab M, et al: Alcohol drinking and pancreatic cancer risk: a metaanalysis of the dose-risk relation. Int J Cancer 2010;126:1474-1486.

6 Lowenfels AB, Maisonneuve P, DiMagno EP, et al: Hereditary pancreatitis and the risk of pancreatic cancer. International Hereditary Pancreatitis Study Group. J Natl Cancer Inst 1997;89:442-446.
7 Amundadottir L, Kraft P, Stolzenberg-Solomon RZ, et al: Genome-wide association study identifies variants in the $\mathrm{ABO}$ locus associated with susceptibility to pancreatic cancer. Nat Genet 2009;41:986-990.

8 Chari ST, Leibson CL, Rabe KG, et al: Probability of pancreatic cancer following diabetes: a population-based study. Gastroenterology 2005;129:504-511.

9 Hruban RH, Boffetta P, Hiraoka N, et al: Ductal adenocarcinoma of the pancreas; in Bosman FT, Carneiro F, Hruban RH, Theise ND, et al (eds): WHO Classification of Tumours of the Digestive System, ed 4. Lyon, IARC, 2010, pp 281-291.

10 Hruban RH, Takaori K, Klimstra DS, et al: An illustrated consensus on the classification of pancreatic intraepithelial neoplasia and intraductal papillary mucinous neoplasms. Am J Surg Pathol 2004;28:977-987.

11 Hruban RH, van Mansfeld AD, Offerhaus GJ, et al: K-ras oncogene activation in adenocarcinoma of the human pancreas. A study of 82 carcinomas using a combination of mutantenriched polymerase chain reaction analysis and allele-specific oligonucleotide hybridization. Am J Pathol 1993;143:545-554.
12 DiGiuseppe JA, Hruban RH, Goodman SN, et al: Overexpression of p53 protein in adenocarcinoma of the pancreas. Am J Clin Pathol 1994;101:684-688.

13 Boschman CR, Stryker S, Reddy JK, et al: Expression of $\mathrm{p} 53$ protein in precursor lesions and adenocarcinoma of human pancreas. Am J Pathol 1994;145:1291-1295.

14 Caldas C, Hahn SA, da Costa LT, et al: Frequent somatic mutations and homozygous deletions of the p16 (MTS1) gene in pancreatic adenocarcinoma. Nat Genet 1994;8:2732.

15 Schutte M, Hruban RH, Geradts J, et al: Abrogation of the $\mathrm{Rb} / \mathrm{p} 16$ tumor-suppressive pathway in virtually all pancreatic carcinomas. Cancer Res 1997;57:3126-3130.

16 Geradts J, Hruban RH, Schutte M, et al: Immunohistochemical p16INK4a analysis of archival tumors with deletion, hypermethylation, or mutation of the CDKN2/MTS1 gene. A comparison of four commercial antibodies. Appl Immunohistochem Mol Morphol 2000;8:71-79.

17 Hahn SA, Schutte M, Hoque ATMS, Moskaluk CA, et al: DPC4, a candidate tumor suppressor gene at human chromosome18q21.1. Science 1996;271:350-353. 
18 Hahn SA, Hoque ATMS, Moskaluk CA, et al: Homozygous deletion map at 18q21.1 in pancreatic cancer. Cancer Res 1996;56:490-494.

19 Schutte M, Hruban RH, Hedrick L, et al: DPC4 gene in various tumor types. Cancer Res 1996;56:2527-2530.

20 Wilentz RE, Su GH, Dai JL, et al: Immunohistochemical labeling for dpc4 mirrors genetic status in pancreatic adenocarcinomas: a new marker of DPC4 inactivation. Am J Pathol 2000;156:37-43.

21 Jones S, Zhang X, Parsons DW, et al: Core signaling pathways in human pancreatic cancers revealed by global genomic analyses. Science 2008;321:1801-1806.

22 Biankin AV, Waddell N, Kassahn KS, et al: Pancreatic cancer genomes reveal aberrations in axon guidance pathway genes. Nature 2012;491:399-405.

23 Witkiewicz AK, McMillan EA, Balaji U, et al: Whole-exome sequencing of pancreatic cancer defines genetic diversity and therapeutic targets. Nat Commun 2015;6:6744.

24 Hruban RH, Wilentz RE, Kern SE: Genetic progression in the pancreatic ducts. Am J Pathol 2000;156:1821-1825.
25 Yanagisawa A, Ohtake K, Ohashi K, et al: Frequent $\mathrm{c}-\mathrm{Ki}$-ras oncogene activation in $\mathrm{mu}$ cous cell hyperplasias of pancreas suffering from chronic inflammation. Cancer Res 1993; 53:953-956.

26 Wilentz RE, Geradts J, Maynard R, et al: Inactivation of the p16 (INK4A) tumor-suppressor gene in pancreatic duct lesions: loss of intranuclear expression. Cancer Res 1998;58: 4740-4744.

27 Wilentz RE, Iacobuzio-Donahue CA, Argani $\mathrm{P}$, et al: Loss of expression of Dpc4 in pancreatic intraepithelial neoplasia: evidence that DPC4 inactivation occurs late in neoplastic progression. Cancer Res 2000;60:2002-2006.

28 Oda Y, Aishima S, Morimatsu K, et al: Pancreatic intraepithelial neoplasia in the background of invasive ductal carcinoma of the pancreas as a prognostic factor. Histopathology 2014;65:389-397.

29 Oshima M, Okano K, Muraki S, et al: Immunohistochemically detected expression of 3 major genes (CDKN2A/p16, TP53, and SMAD4/DPC4) strongly predicts survival in patients with resectable pancreatic cancer. Ann Surg 2013;258:336-346.
30 Baas IO, Mulder JW, Offerhaus GJ, et al: An evaluation of six antibodies for immunohistochemistry of mutant p53 gene product in archival colorectal neoplasms. J Pathol 1994; 172:5-12.

31 Melhem MF, Law JC, el-Ashmawy L, et al: Assessment of sensitivity and specificity of immunohistochemical staining of p53 in lung and head and neck cancers. Am J Pathol 1995; 146:1170-1177.

32 Hassid BG, Lucas AL, Salomao M, et al: Absence of pancreatic intraepithelial neoplasia predicts poor survival after resection of pancreatic cancer. Pancreas 2014;43:1073-1077.

33 Vang R, Ie Shih M, Kurman RJ: Ovarian lowgrade and high-grade serous carcinoma: pathogenesis, clinicopathologic and molecular biologic features, and diagnostic problems. Adv Anat Pathol 2009;16:267-282.

34 George SM, Mäkinen MJ, Jernvall P, et al: Classification of advanced colorectal carcinomas by tumor edge morphology: evidence for different pathogenesis and significance of polypoid and nonpolypoid tumors. Cancer 2000;89:1901-1909.
Molecular Characteristics of PDACs with and without High-Grade PanIN
Pathobiology 2017;84:192-201 